
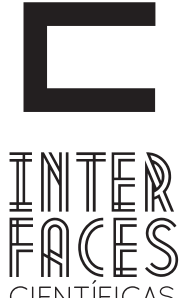

HUMANASE SOCIAIS

\title{
DOENÇA DE CHAGAS INFANTO JUVENIL EM ÁREA RURAL DO NORDESTE BRASILEIRO: RISCO DE TRANSMISSÃO E REFLEXÕES SOCIAIS
}

Weber de Santana Teles ${ }^{1}$

Kleber Willer Coutinho de Santana ${ }^{3}$

Veronica de Lourdes Sierpe Jeraldo ${ }^{5}$

\author{
Maria Hozana Santos Silva² \\ Rubens Riscala Madi ${ }^{4}$ \\ Claudia Moura de Melo ${ }^{6}$
}

\section{RESUMO}

A doença de Chagas infantil no Brasil tem apresentando um padrão de redução na soroprevalência em áreas sob o impacto de diferentes tempos de intervenção de controle. Estas intervenções, no entanto, são descontínuas e algumas vezes negligenciadas. 0 objetivo deste estudo foi avaliar o risco de infecção infantil da doença de Chagas e delinear seus principais reflexos sociais em área rural endêmica, localizada no município de Itabaianinha/SE. O estudo foi realizado em 198 indivíduos na faixa etária de 1-14 anos de ambos os sexos, sem distinção étnica. Foram aplicados aos pais ou responsáveis pelas crianças o questionário utilizado pelo Programa de Controle da Doença de Chagas, contendo informações sobre variáveis como gênero, faixa etária, grau de escolaridade, contato com triatomíneo, sintomas, entre outras. Paralelamente foram coletadas amostras de sangue das crianças e submetidas às técnicas de ELISA e imunofluorescência indireta. Das 198 crianças pesquisadas, $51,5 \%$ eram do sexo masculino e $48,5 \%$, do sexo feminino. Sobre as variáveis de risco, $31.4 \%$ dos indivíduos relatam ter visto barbeiros no domicilio e $8.1 \%$ dizem tê-lo manipulado, $0.6 \%$ já realizaram transfusão sanguínea, $4.6 \%$ moram com algum chagásico, $70.7 \%$ possuem animal doméstico e $25,3 \%$ moram em habitação de pau a pique. Nenhuma das amostras submetidas à avaliação sorológica apresentou reatividade. As informações obtidas permitem um melhor entendimento dos aspectos sociais envolvidos no risco de transmissão do Trypanosoma cruzi que poderão contribuir para o desenvolvimento de efetivas estratégias de controle da doença de Chagas na região de Itabaianinha/SE.

\section{PALAVRAS-CHAVE}

Tripanossomíase Americana. Epidemiologia. Condições Sociais. 


\section{ABSTRACT}

Chagas disease in children presenting Brazil has a standard reduction in seroprevalence in areas under the impact of different intervention times control. These measures, however, are discontinuous and sometimes neglected. The aim of this study was to evaluate the risk of infant infection of Chagas disease and outline its main social reflexes in endemic area, located in the municipality of Itabaianinha / SE. The study was conducted on 198 individuals aged 1-14 years of both sexes, without ethnic distinction. Were administered to parents or guardians of children the questionnaire used by Chagas Disease Control Program containing information on variables such as gender, age, educational level, contact with triatomine, symptoms, among others. Parallel blood samples from the children were collected and subjected to ELISA and indirect immunofluorescence. Of the 198 children sur-

\section{RESUMEN}

La enfermedad de Chagas en niños en Brasil, viene mostrando un patrón de reducción de seroprevalencia en regiones bajo impacto de medidas de intervención en diferentes tiempos. Estas medidas, sin embargo, son discretas y, a veces olvidadas. El objetivo de este estudio fue evaluar el riesgo de infección en los niños con la enfermedad de Chagas y esbozar sus principales reflexiones sociales en una zona endémica del municipio de Itabianinha, estado de Sergipe/Brasil. El estudio se realizó en 198 individuos de 1 a 14 años de ambos sexos, sin distinción étnica. A los padres o tutores, se les aplicó el cuestionario utilizado por el Programa de Control de la Enfermedad de Chagas que contiene informaciones sobre variables como género, edad, nivel educativo, contacto con triatominos (vinchucas), síntomas, entre otros. Paralelamente, se recogieron muestras de sangre de los niños que fueron sometidas a las técnicas de ELISA y de inmunofluorescencia indirecta. De los 198 veyed, $51.5 \%$ were male and $48.5 \%$ female. About the risk variables, $31.4 \%$ of individuals report seeing barbers at home and $8.1 \%$ say they have it handled, $0.6 \%$ had conducted blood transfusion, $4.6 \%$ live with some chagasic, $70.7 \%$ have a household pet and $25.3 \%$ live housing of wattle and daub. None of the samples submitted for serologic evaluation showed reactivity. The obtained data allow a better understanding of the social aspects involved in the risk of transmission of Trypanosomacruzi that may contribute to the development of effective strategies for the control of Chagas disease in the region of Itabianinha/SE.

\section{KEYWORDS}

American Tripanosomiasis. Epidemiology. Social Conditions.

niños encuestados, el 51,5\% eran hombres y $48,5 \%$ mujeres. En relación a las variables de riesgo, el 31,4 $\%$ de los sujetos declararon haber visto vinchucas en sus casas y el 8,1\% dicen haberlas manipulado, 0.6 $\%$ ya realizaron transfusión de sangre, el 4,6 \% vive con un familiar chagásico, 70,7 \% tienen mascotas y el $25,3 \%$ habita en viviendas construidas con madera e barro. Ninguna de las muestras serológicas mostró reactividad. Los datos obtenidos permiten una mejor comprensión de los aspectos sociales involucrados en el riesgo de transmisión de Trypanosoma cruzi que pueden contribuir al desarrollo de estrategias efectivas para el control de la enfermedad de Chagas en la región de Itabianinha/SE.

\section{PALABRAS CLAVE}

Tripanosomiasis americana. Epidemiología. Condiciones sociales. 


\section{INTRODUÇ̄̃O}

A doença de Chagas (DC) é uma endemia com prevalência em torno de 16 a 18 milhões de indivíduos nos diversos países do continente americano. Originalmente rural, está associada a fatores ambientais, sociais e políticos em que os indivíduos mais afetados são aqueles que vivem em condições precárias de habitação, o que favorece a presença dos insetos vetores, conhecidos popularmente como barbeiros, nos seus domicílios e peridomicílios (DIAS, 2011).

Devido à dimensão continental da endemia, o médico e cientista brasileiro Carlos Chagas jamais deixou de abordar a urgência e necessidade do controle. Diante deste panorama, Chagas relatava às autoridades sanitárias e políticas da época o impacto social da doença, mostrando as suas graves consequências e apontando ações técnicas e político-sociais que deveriam ser executadas, norteadas pela dispersão da DC e sua relação com a precariedade de moradia na zona rural (DIAS, 2011).

Desde sua descoberta, a DC constituiu-se numa endemia predominantemente rural no continente americano, atingindo áreas específicas, intimamente associadas ao subdesenvolvimento social e econômico. Somente nas ultimas décadas do século XX, seu padrão epidemiológico passou por uma mudança devido ao êxodo rural, tonando-a uma endemia urbano-rural (RAMOS et al., 2012).

Na América Latina, a DC é considerada uma das principais doenças negligenciadas, e a de quarto maior impacto social (DIAS, 2001). A prevalência gira em torno de $1,4 \%$, no entanto tal estimativa pode não refletir a real situação, devido às dificuldades encontradas na obtenção de registros nos serviços de vigilância e/ou atendimento e a baixa procura por atendimento médico pela população acometida (CORTES et al., 2012).

Segundo dados da Organização Mundial de Saúde (WHO, 2012), a estimativa da infecção seria de 16 a 18 milhões de pessoas de 18 países americanos. No
Brasil, estima-se que a área de abrangência da DC se estenda desde o estado do Maranhão até o Rio Grande de Sul, com dois a três milhões de pessoas infectadas (MINISTÉRIO DA SAÚDE, 2012).

O impacto social da DC é expressivo, uma vez que se estima que de 10 a 40\% dos indivíduos infectados já têm ou terão uma cardiopatia crônica devida à infecção. Além da cardiopatia, as disfunções digestivas, absenteísmo, custos previdenciários e médico-hospitalares, processos de perpetuação de pobreza familiar em zonas endêmicas, baixa produtividade e altos custos de programas de controle e vigilância são fatores que implicam em importantes gastos financeiros e sociais dos países sul-americanos afetados pela endemia (DIAS, 2007).

No Nordeste brasileiro a DC ocupa, no contexto epidemiológico, acentuada importância, tendo sido apontado como a segunda parasitose em quantidade de indivíduos infectados, com uma prevalência de $3,1 \%$. Por outro lado, a região apresenta os maiores índices de habitações humanas de baixa qualidade e com condições ambientais/ estruturais adequadas para atuar como abrigo para os vetores, o que perpetua a endemia na região (DIAS, 2000).

Os estados da Bahia e Sergipe são as áreas com maiores taxas de prevalência, alcançando $5,4 \%$ e $6 \%$ respectivamente. Essa região brasileira vem apresentando grande importância epidemiológica por configurar-se na segunda área quanto à quantidade de indivíduos infectados e de índices de infestação triatomínica (BRITO et al., 2012).

No estado de Sergipe a DC pode ser considerada um relevante problema de saúde, especialmente em sua área rural, uma vez que esta apresenta condições de natureza antrópica e político sociais propícias para a multiplicação dos insetos vetores, tais 
como: habitações rurais de má qualidade, populações socialmente excluídas, baixo investimento em vigilância sanitária e epidemiológica, espaços geograficamente abertos, além de um crescente desequilíbrio ambiental.

Em Sergipe, $12 \%$ dos seus municípios estão classificados como áreas de alto risco para transmissão da DC (SES/SE 2011), incluindo-se Itabaianinha, alvo do presente estudo. Apesar do reconhecimento da DC como problema de saúde pública, pouco se sabe em relação à dinâmica e risco de transmissão de doença na população infantil. Dessa forma, o objetivo desta pesquisa foi realizar um estudo, com intuito de avaliar aspectos sorológicos e socioepidemiológicos em relação ao risco de transmissão da doença de Chagas em população infantil de área rural endêmica para DC do estado de Sergipe.

\section{METODOLOGIA}

\section{1 ÁREA DE ESTUDO}

O município de Itabaianinha se localiza no sul-sergipano, possui área territorial de 480,4 Km2. A população estimada é de 35.123 habitantes sendo que $15.572(43,8 \%)$ se encontram na zona urbana e $19.571(55,72 \%)$ na zona rural. O perfil econômico está voltado para a plantação de frutas cítricas, criação de animais e de fábricas de material de construção (telhas e blocos) (IBGE, 2011).

\subsection{POPULAÇÃOO}

A população em estudo foi constituída de todas as crianças na faixa etária de 0-14 anos residentes nos povoados de Itabaianinha que apresentaram casos de doença de Chagas aguda notificados em 2012 (Tabela 1). Desta forma 4 foram os povoados escolhidos para realização da pesquisa: Mutuca, Piabas, Fundão e Água Boa.
Tabela 1 - Distribuição da população total e de crianças na faixa etária de 0-14 anos por povoado e número de casos notificados de doença de Chagas no município de Itabaiana/SE em 2012.

\begin{tabular}{lcc}
\hline \multicolumn{1}{c}{$\begin{array}{c}\text { Povoado } \\
\text { (população total) }\end{array}$} & \multicolumn{2}{c}{ Casos de Doença de Chagas } \\
& Crianças & $\mathbf{( \% )}$ \\
\hline Mutuca (201) & 53 & $2(0,99)$ \\
Piabas (334) & 106 & $1(0,27)$ \\
Fundão (448) & 126 & $1(0,27)$ \\
Água Boa (368) & 79 & $1(0,27)$ \\
Total (1.351) & $\mathbf{3 6 4}$ & $\mathbf{5 ( 0 , 3 7 )}$ \\
\hline
\end{tabular}

A sensibilização da comunidade foi realizada por meio de metodologia participativa em que as estratégias de desenvolvimento do estudo foram definidas entre os pesquisadores e comunidade. Inicialmente foram realizadas palestras de sensibilização em ambiente escolar em cada um dos povoados, com a participação dos pais/responsáveis das crianças, alunos, professores e dos técnicos da Coordenação de Endemias. Criou-se, então, um espaço de troca de experiências sobre a DC com enfoque nas formas de infecção, agente transmissor, práticas de higiene domiciliar, entre outras. Aos pais/responsáveis pelas crianças que concordaram em participar da pesquisa foi aplicado o questionário utilizado pelo Programa de Controle da Doença de Chagas (PCDCh) e que compila informações de saúde, moradia, aspectos socioeconômicos, entre outros.

\subsection{ASPECTOSÉTICOS}

O estudo foi realizado seguindo as normas éticas constantes na resolução do CNS 196/96, sendo aprovado pelo comitê de ética da Universidade Tiradentes, sob o protocolo $n^{0} 070309$.

\subsection{AVALIAÇ̃̃O SOROLÓGICA}

Para avaliação sorológica foram colhidos $10 \mathrm{ml}$ de sangue por punção venosa das crianças que juntamente aos seus responsáveis se apresentaram de forma voluntária. 
A soropositividade para infecção chagásica foi definida a partir do resultado confirmatório de dois testes aplicados: sorológico (primeira escolha - ELISA) e confirmatório (imunofluorescência) (MINISTÉRIO DA SAÚDE, 2005).

Foi realizada análise descritiva dos dados numéricos, com os resultados expostos em Tabelas de frequência.

\section{RESULTADOS}

Os povoados de Itabaianinha estudados abrigam uma população infanto juvenil de 364 indivíduos na faixa etária de 0-14 anos, das quais 60\% (198) participaram do inquérito e da avaliação sorológica. Dentre as crianças avaliadas, 51,5\% (102) foram do sexo masculino e $48,5 \%$ (96) do sexo feminino.

Em relação aos indicadores socioeconômicos, o nível de escolaridade predominante dos pais/responsáveis é o ensino fundamental incompleto (96\%); 93,4\% (185) tinham abastecimento de água oriundo de poço e 6.6\% (13) de água encanada; destes, 1,01\% afirmaram que a água de consumo era tratada por meio de fervura, enquanto 98,9\% afirmaram utilizar água diretamente da fonte sem nenhum tipo de tratamento. Quanto ao saneamento ambiental, 66\% $(n=132)$ dos pais/responsáveis relataram queimar o lixo, 32,9\% $(n=65)$ o enterram e $0,5 \%(n=1)$ utilizam o serviço de coleta público.

Ao avaliar-se a ocorrência de sinais ou sintomas relacionados à doença de Chagas, os pais/responsáveis apontaram febre, mal-estar, inchaço ocular e falta de apetite (15,7\%), enquanto 84,3\% não relataram nenhum. Em relação ao acesso à saúde, $89,3 \%(n=177)$ dos indivíduos entrevistados consideram-no escasso e difícil.

Quanto à infraestrutura habitacional, 74,7\% ( $\mathrm{n}=148)$ das crianças residem em casa de alvenaria, sendo que, $25,3 \%(n=50)$ moram em casas de pau a pique. Aproximadamente $60 \%(n=121)$ destas unidades domiciliares apresentam energia elétrica. Em relação ao risco potencial de infecção por vias vetorial e oral, 68,6\% (136) re- latam ter observado o barbeiro no interior da residência, sendo que destes 8,1\% (16) relataram tê-lo manipulado. Em relação à presença de animais domésticos no domicílio, 70,7\% (140) afirmam possuir (cães e gatos).

O nível de conhecimento sobre a doença de Chagas dos indivíduos da área rural de Itabaianinha é muito baixo uma vez que os pais/responsáveis relataram nunca terem recebido informações a respeito da doença por parte de profissionais de saúde e, portanto não sabiam quais as formas de prevenção. Somente um dos pais relatou saber como se contrai a doença e 2,6\% (5) afirmaram conhecer o barbeiro, embora 9 deles $(4,6 \%)$ relatem ter um ou mais membros da família com diagnóstico positivo para DC, motivo pelo qual mostraram preocupação e interesse em conhecer melhor a DC. Em relação ao risco associado à ingestão de caldo de cana e consequentemente do parasita por via oral, $14,2 \%(n=28)$ relatam que as crianças já ingeriram esta bebida em alguma oportunidade.

A avaliação sorológica não revelou reatividade em nenhuma das 198 amostras sanguíneas, mostrando que à data da coleta não existiam crianças infectados com o parasita.

\section{DISCUSSÃO}

Segundo a Organização Panamericana de Saúde, o grupo etário abaixo de 5 anos é considerado o mais indicado para estudos de soroprevalência que avaliem as condições de transmissão recente da doença de Chagas em um determinado local (OSTERMAYER et al., 2011). 0 presente estudo ampliou a faixa etária a ser avaliada com o intuito de ampliar a adesão dos pais/responsáveis para realização do teste sorológico das crianças, uma vez que o deslocamento nas estradas de má qualidade é difícil, especialmente nos períodos chuvosos. Devido à falta de serviço de transporte público regular, os moradores locomovem-se a pé ou por meio de ciclomotores e bicicletas, o que dificulta o acesso aos serviços de saúde. 
A soronegatividade na população infantil estudada pode ser reflexo da diminuição da transmissão vetorial que pode ser resultante da eficácia das ações regulares do PCDCh de Itabaianinha, único programa atuante no estado de Sergipe. No Brasil, situação epidemiológica semelhante foi observada em estudo realizado em 2007, com crianças na faixa etária de 0 a 5 anos, totalizando 94 mil amostras de sangue coletadas, no qual também foi encontrada uma soroprevalência da DC de 0\% (MONCAYO; SILVEIRA, 2009). No Chile, em pesquisa realizada em 2005 em 1.088 crianças menores de 10 anos procedentes da zona rural, também não foram detectados casos positivos da doença, indicando uma possível interrupção da transmissão vetorial da DC (LORCA et al., 2006).

No território brasileiro a soropositividade infantil para DC tem apresentado tendência de redução. Borges-Pereira e outros autores (2002), realizando investigação no Piauí, Brasil, detectaram soropositividade de $9,8 \%$ na população total e de $0,5 \%$ em menores de 10 anos. Em pesquisa realizada em Rio Grande do Norte, somente foram encontrados casos positivos para DC acima dos 18 anos, mostrando que a soropositividade parece aumentar de forma significativa com a idade (BRITO e col., 2012). No Ceará, estudo transversal (BORGES-PEREIRA et al., 2002) determinou soroprevalência da infecção chagásica de $3,1 \%$, maior entre as pessoas com mais de 50 anos. 0 presente estudo, realizado em Itabaianinha/Se, configura-se no primeiro mapeamento de soroprevalência infantil para DC em área endêmica de Sergipe.

0 recorte populacional homogêneo de meninas e meninos itabaianenses apresentaria o mesmo grau de risco de adquirir a DC, levando-se em consideração o caráter sexo isoladamente, uma vez que não há correlação entre os sexos masculino e feminino em relação à soropositividade (BRITO et al., 2012). No entanto, existe um risco potencial, mesmo no atual cenário de soronegatividade, em função dos casos notificados (Tabela 1) e do índice de infecção natural dos triatomíneos vetores da ordem de 31,3\% (LIMA et al., 2012). Neste contexto, avaliações de custo-benefício ou custo-efetividade já têm mostrado que o controle das condições de risco de transmissão da DC é muito mais efetivo que os gastos com atenção ao paciente chagásico (DARIUSH, 1998).

Em estudo da relação custo-efetividade de tratamento da doença de Chagas, Saggia e outros autores (2007) afirmam que o custo total médio por paciente chagásico em tratamento específico é de US\$ 674,87 , enquanto que o custo total médio/ano para paciente em tratamento não específico eleva-se para US\$1.116,63. Uma população infantil sob risco de infecção para DC pode ser responsável por custos econômicos maiores devido ao seu maior tempo de vida médio, aliado ainda a formação de bolsões de baixa produtividade devido a perda da capacidade de trabalho dos adultos jovens. Os custos indiretos como perda de produtividade e aposentadorias precoces relacionadas à doença não foram incluídos.

De acordo com o Ministério da Saúde (2002), o indivíduo chagásico compõe um segmento populacional de baixo nível socioeconômico e social, excluído pela conjunção de pobreza e doença, o que the confere pouco poder de reivindicação e praticamente nenhuma visibilidade política. As evidências indicam que as condições socioeconômicas estão relacionadas ao processo saúde-doença, como renda, educação, emprego e capacidade de exercer a cidadania.

$\mathrm{Na}$ área sergipana estudada, a grande maioria dos entrevistados tem baixo nível de escolaridade, o que sem dúvida pode se refletir em carência no conhecimento/ entendimento do processo saúde-doença e seus determinantes/condicionantes, e no menor cuidado da população infantil/jovem com relação a DC. O pouco conhecimento dos pais/responsáveis tem caráter empírico e foi obtido por meio da transmissão de saberes/experiências de indivíduos chagásicos familiares ou conhecidos.

Apesar de várias pesquisas relatarem que indivíduos chagásicos viram o triatomíneo no interior do domicílio (BRITO et al., 2012), como relatado, 
também, nesta pesquisa, é necessário uma avaliação crítica com relação a estes dados em função dificuldade de identificação do barbeiro.

Durante o desenvolvimento das entrevistas foi possível identificar a falta de informações quanto aos mecanismos de transmissão, as formas clínicas da doença, bem como seus respectivos sintomas, terapêutica e prevenção, isto seria reflexo da ausência de ações de educação em saúde. Os escassos relatos de ocorrência de sintomas por parte dos pais/responsáveis (febre, inchaço ocular, mal estar e falta de apetite) são inespecíficos e parecem estar relacionados ao cuidado infantil mal ou não realizado, devido à dificuldade de acesso aos serviços de saúde. A falta de percepção que os indivíduos apresentam quanto à sua limitação cotidiana expressa um processo de alienação; desta forma, os indivíduos precisam enfrentar seu processo de adoecimento para participar efetivamente do seu cuidado.

A DC é de origem silvestre e em decorrência das modificações ambientais como os desmatamentos não planejados, uso de agrotóxicos e as condições sociais como precariedade habitacional e de higiene, decorrentes do processo histórico brasileiro, possibilitaram o surgimento da "doença humana" e sua perpetuação, como observado na pesquisa (BLACK et al., 2007; DIAS, 2007).

A transmissão vetorial da DC ocorre predominantemente em áreas rurais de baixa densidade populacional (VINHAES; DIAS, 2000), característica demográfica típica dos povoados de Itabaianinha. A distribuição espacial das vivendas rurais desta região mostram que elas distam, em média, aproximadamente $4 \mathrm{~km}$ umas das outras (LIMA et al., 2012). A baixa densidade populacional aliada a grande distância entre as residências pode configurar-se em fator de risco para a infecção chagásica, uma vez que os triatomíneos são insetos gregários e adaptados a locais escuros e calmos.

Moradores da área relatam que os insetos entram nas habitações voando. O início da atividade de vôo curto destes insetos pode ser induzido por fatores am- bientais ou antrópicos, tais como a elevação da temperatura, escassez nutricional (LEHANE et al., 1992) e stress reprodutivo, que em Itabaianinha podem estar relacionas as queimadas e utilização de pesticidas na cultura de laranjas e ao funcionamento das olarias.

Em acréscimo aos determinantes de natureza biológica/ecológica, nos fatores de natureza econômica e social (casas mal construídas, mal acabadas ou mal conservadas e a desinformação), podem ser encontradas as razões da manutenção da transmissão da DC em determinadas áreas. Pesquisa realizada na América Latina identificou tipos de material de construção dos domicílios/peridomicílios como fatores associados à infecção pelo T. cruzi, sendo demonstrada a influência da infraestrutura habitacional na prevalência de infestação triatomínica (BLACK et al., 2007).

Na área rural de Itabaianinha, embora as residências sejam de alvenaria em mais de $74 \%$, os anexos (galinheiro, curral, estábulo, chiqueiro, depósito de ferramentas/produtos agrícolas) são de construção precária (LIMA et al., 2012), servindo de abrigo para animais domésticos, o que seria um fator de risco de transmissão da DC. Na região estudada, embora se observe melhoria das habitações humanas, esta não foi acompanhada de ações educacionais, como orientações sobre o asseio ao interior das habitações e das áreas externas.

Por outro lado, na área rural de Itabaianinha, observa-se processo de adaptabilidade dos insetos vetores em habitações humanas com boa infraestrutura, como as de alvenaria, incluindo-se as casas construídas com recursos públicos como forma de atenuar a transmissão vetorial.

Existem controvérsias com relação ao papel da energia elétrica domiciliar como fator de risco para a transmissão da DC. Embora a luz elétrica possa ser considerada como um dos fatores potenciais relacionados com a ocorrência da doença (FÉ et al., 2009), ressalta-se, por outro lado, uma associação significante entre a presença da eletricidade e a diminuição de colonização das casas por triatomíneos. A maioria da população estudada tem 
acesso a serviço de energia elétrica (60\%), o que poderia ser um fator influenciador da diminuição da colonização peri e intradomiciliar do barbeiro e consequente soronegatividade das crianças.

Conforme Walter (2007), os fatores de riscos para infestação peridomiciliar são principalmente a quantidade de animais domésticos, tais como cães, gatos, aves e mamíferos de pequeno porte e presença de entulhos. Estes animais podem ser carreadores de triatomíneos de locais infestados para aqueles sem infestação, atuando como fonte de alimento para os barbeiros no ambiente peridomiciliar. Em alguns locais, esses animais compartilham com o homem o espaço das habitações humanas, o que eleva o risco de transmissão da DC (RIOS-OSORIO et al., 2012).

Por se tratar de área rural, a maioria dos entrevistados mora em domicílios onde existem animais domésticos ou de criação, o que se configura em um fator de risco local para DC. Por outro lado, os animais domésticos ao caçar em ambientes silvestres podem se tornar infectados, podendo levar infecção para o ambiente domestico ou áreas próximas. Sendo assim, a adaptação dos triatomíneos ao ambiente domiciliar juntamente com a circulação de T. cruzi entre eles e os animais domésticos/silvestres é certamente o mais importante determinante para o estabelecimento da infecção humana (COURA, 2007).

\section{CONCLUSÃO}

A baixa prevalência de doença aguda na área, segundo dados do PCDCh, e a soronegatividade para Doença de Chagas em indivíduos na faixa de 0-14 anos pode ser indicador de redução da transmissão vetorial. No entanto, é importante ressaltar que muitas das condições consideradas de risco como o baixo nível de escolaridade, saneamento ambiental precário, acesso à saúde deficiente, presença de barbeiros e de animais domésticos, bem como o desconhecimento de aspectos relacionados à transmissão e prevenção da DC, levam a pensar na necessidade de que ações de controle devam ser mantidas e intensificadas, assim como o monitoramento das crianças e adultos da região.

\section{REFERÊNCIAS}

BLACK, C.L.; OCAÑA, S.; RINER, D.; COSTALES, J.A.; LASCANO, M.S.; DAVILA, S.; ARCOS-TERAN, L.; SEED, J.R.; GRIJALVA, M.J. Household risk factors for Trypanosoma cruzi seropositivity in two geographic regions of Ecuador. J. Parasitol., v.93, n.1, 2007, p.12-16.

BORGES-PEREIRA, P.J.; CASTRO, J.A.F.; CAMPOS, J.H.F.; NOGUEIRA, J.S.; ZAUZA, P.L.; MARQUES, P.; CARDOSO, M.A.; BRITTO, C.; ARAUJO, A.J.G. Estudo da infecção e morbidade da doença de Chagas no município de João Costa: Parque Nacional Serra da Capivara, Piauí, Brasil. Rev. Soc. Bras. Med. Trop., v.35, n.4, 2002, p.315-322.

BRITO, C.R.N.; SAMPAIO, G.H.F.; CÂMARA, A.C.J.; NUNES, D.F.; AZEVEDO, P.R.M.A.; CHIARI, E.; GALVÃO, L.M.C. Seroepidemiology of Trypanosoma cruzi infection in the semiarid rural zone of the State of Rio Grande do Norte, Brazil. Rev. Soc. Bras. Med. Trop., v.45, n.3, 2012, p.346-352.

CORTEZ, J.; RAMOS, E.; VALENTE, C.; SEIXAS, J.; VIEIRA, A. A expressão global da doença de Chagas - Oportunidades emergentes e impacto em Portugal.

Acta Med. Por., v.25, n.5, 2012, p.332-339.

COURA, J. R. Chagas disease: What is known and what is needed a backgraound article. Mem. Inst. Oswaldo Cruz, v.102, supl. 1, 2007, p.113-122.

DARIUSH. A. Análise do custo - efetividade do programa de controle da doença de Chagas no Brasil: relatório final. Brasilia: Organização Panamericana da Saúde, 1998, 271p.

DIAS J.C.P.D. Educação e participação popular. Participação, descentralização e controle de endemias no 
Brasil. In: BARATA, R.B.; BRICEÑO-LEÓN, R. (Orgs.). Doenças endêmicas: abordagens sociais, culturais e comportamentais. Rio de Janeiro: Fiocruz, 2000.

DIAS, J.C.P.O. Globalização, Iniquidade e Doença de Chagas. Cad. Saúde Pública, v.23, supl.1, 2007, p.S13-S22.

DIAS, J.C.P. Os primórdios do controle da doença de Chagas (em homenagem a Emmanuel Dias, pioneiro do controle, no centenário de seu nascimento). Rev. Soc. Bras. Med. Trop., v.44, supl.2, 2011, p.12-18.

DIAS, J.P.C. Doença de Chagas, ambiente, participação e Estado. Cad. Saúde Pública, v.17, 2001, p.165-169.

FÉ, N.F.; MAGALHÃES, L.K.; FÉ, F.A.; ARAKIAN, S.K.; MONTEIRO, W.M.; BARBOSA, M.G.X. Ocorrência de triatomíneos em ambiente silvestre e domiciliares no município de Manaus estado do Amazonas. Rev. Soc. Bras. Med. Tropical, v.46, n.6, 2009, p.642-646.

IBGE - INSTITUTO BRASILEIRO DE GEOGRAFIA E ESTATÍSTICA. Disponível em: <http://www.ibge.gov.br/ cidadesat/link.php?uf=se>. Acesso em: 5 dez. 2013.

LEHANE, M.J.; MCEWAN, P.K.; WHITAKER, C.J.; SCHOFIELD, C.J. The role of temperature and nutritional status in flight initiation by Triatoma infestans. Acta Trop., v.52, n.1, 1992, p.27-38.

LIMA. A.F.R; JERALDO, V.L.S.; SILVEIRA, M.S.; MADI, R.R.; SANTANA, T.B.K.; MELO, C.M. Triatomines in dwellings and outbuildings in an endemic area of Chagas disease in northeastern Brazil. Rev. Soc. Bras. Med. Trop., v.45, n.6, 2012, p.701-706.

LORCA, M.; SOTO, P.; RUIZ, M.; CONTRERAS, M.C.; SALINAS, P.; GUERRA, A.; IRRIBARRA, N.; BODOR, P.; VIVANCO, P.; ARREDONDO, A.; TRINCADO, A.; RAPHAEL, T.; MILTON EGAÑA, M.; CARLOS PAVLETIC, C.; ALONSO PARRA, A. Estudio serológico para enfermedad de Chagas en menores de 10 años em Valparaíso y San Antonio, Chile. Rev. Méd. Chile, v.134, 2006, p.1345-1346.
MINISTÉRIO DA SAÚDE. Secretaria de Vigilância em Saúde. Guia de Política Nacional de Promoção de Saúde. Brasília: Ministério da Saúde, 2002, 60p.

MINISTÉRIO DA SAÚDE. Consenso Brasileiro em Doença de Chagas. Rev. Soc. Bras. Med. Trop., v.38, supl. III, 2005, p.1-30.

MINISTÉRIO DA SAÚDE. Secretaría de Vigilância em Saúde. Bol. Epidemiol., v.43, 2012, p.1-16.

MONCAYO, A.; SILVEIRA, A.C. Current epidemiological trends for Chagas disease in Latin America and future challenges in epidemiology, surveillance and health policy. Mem. Inst. Oswaldo Cruz, v.104, 2009, p.17-30.

OSTERMAYER, A.L.; PASSOS, A.D.C.; SILVEIRA, A.C.; FERREIRA, A.W.; MACEDO, V.; PRATA, A.R. O inquérito nacional de soroprevalência de avaliação do controle da doença de Chagas no Brasil (2001-2008). Rev. Soc. Bras. Med. Trop., v.4, sup.2, 2011, p.108-121.

RAMOS, J.M.; PONCE, J.; GALLEGOS. I.; FLORES-CHAVEZ, M.; CAÑAVATE, C.; GUTIERREZ, F. Trypanosoma cruzi infection in Elche (Spain): comparison of the seroprevalence in immigrants from Paraguay and Bolivia. Path. Global Health, vol.106, n.2, 2012, p.102106.

RIOS-OSORIO, L.A.; CRESPO-GONZÁLEZ, J.; ZAPATA-TAMAYO, M.A. Etnometodología para la comprensión y el manejo de la enfermedad de Chagas en las poblaciones indígenas Wiwa asentadas en la vertiente suroriental de la Sierra Nevada de Santa Marta. Saúde Soc., v.21, n.2, 2012, p.446-457.

SAGGIA, M. G.; SANTOS, E. A. V.; DIETZE, R. Custo-efetividade de benzonidazol para a doença de Chagas no Brasil. In: VIII Encontro ABRES, 2007, São Paulo.

Caderno de Resumos do Encontro da Associação Brasileira de Economia da Saúde, 2007. Disponível em: <http://143.54.230.147/abres/site/arquivos/anais. php\#>. Acesso em: 24 jan. 2014. 
SES/SERGIPE - COORDENAÇÃO DE VIGILANCIA SANITÁRIA. Relatório Técnico do Programa de Controle da Doença de Chagas no estado de Sergipe. Aracaju-SE: Secretaria de Estado da Saúde, 2011.

VINHAES, M. C.; DIAS, J. C. P. Doença de Chagas no Brasil. Cad. Saúde Pública, v.16, 2000, p.7-12.
WALTER, A. Peridomiciliary habitat and risck factors for Triatoma infestation in a rural community of the Mexican Occident. Am. J. Trop. Med. Hyg., v.76, n.3, 2007, p.508-515.
1. Biomédico. Assessor de qualidade e biossegurança no Centro de Hemoterapia de Sergipe - Hemose. E-mail: weber.teles@fsph.se.gov.br

2. Graduanda de Enfermagem da Universidade Tiradentes. E-mail: hozanapatricio@gmail.com

3. Biomédico no Centro de Hemoterapia de Sergipe - Hemose. E-mail: kleber.willer@hotmail.com

4. Doutor em Parasitologia, docente permanente do Curso de Mestrado em Saúde e Ambiente da Universidade Tiradentes. E-mail: rrmadi@ gmail.com

5. Doutora em Parasitologia, docente permanente do Curso de Mestrado em Saúde e Ambiente da Universidade Tiradentes. E-mail: veronica_sierpe@hotmail.com

6. Doutora em Parasitologia, docente permanente do Curso de Mestrado em Saúde e Ambiente da Universidade Tiradentes. E-mail: claudiamouramelo@hotmail.com 